\title{
CONTRIBUTIONS TO THE FILTERING OF NARROWBAND INTERFERENCES IN GNSS
}

\author{
As. Dr. Alexandru RUSU-CASANDRA ${ }^{1}$ \\ Adj. Prof. Dr. Elena-Simona LOHAN ${ }^{2}$ \\ Prof. Dr. Gonzalo SECO-GRANADOS ${ }^{3}$ \\ ${ }^{1}$ Dept. of Telecommunications, University Politehnica of Bucharest, Romania \\ 2 Dept. of Electronics and Communications Engineering, Tampere University of Technology, \\ Finland \\ ${ }^{3}$ Dept. of Telecommunications and System Engineering, Universitat Autonoma de Barcelona, \\ Spain
}

\begin{abstract}
Nowadays, using the services provided by Global Navigation Satellite Systems (GNSS) is ubiquitous in geodesy, cadastre and Geographic Information Systems. Galileo, the GNSS system which is under development by the European Union, will transmit navigation signals in four subbands (E5a/L5, E5b, E6 and E1/L1) within the L band. GNSS systems are not the only users of the L band around the world; other telecommunication systems also use it. Papers analyzing real RF measurement campaigns have shown that loss-of-lock on the navigational signals is possible to GNSS receivers in the vicinity of radio and TV broadcast transmitters. It has also been demonstrated that, in the nearby area of airports, radionavigation or communication systems (e.g. Very High Frequency Omindirectional Radio Range or Instrument Landing System) can cause interference to GNSS receivers. These interferences are unwanted because they can affect the precision of the GNSS receivers and can compromise the topographical measurements. Therefore it is essential to understand better the negative effects of RF interferences on GNSS receivers and to optimize the solutions against the interferences. This paper deals with the particular matter of filtering the narrowband interferences affecting Multiplexed-Binary-Offset-Carrier (MBOC) Galileo navigation signal.
\end{abstract}

Keywords: Global Navigation Satellite Systems, narrowband interferences

\section{INTRODUCTION}

Presently, GNSS systems like European Union's Galileo operate in the IEEE L frequency band, which is located approximately from 1 to $2 \mathrm{GHz}$. [1]. Other telecommunication systems that share the L band are enumerated next [2]: aeronautical navigation systems like civilian Distance Measuring Equipment (DME); air traffic control radars; military and government systems for terrestrial communication, navigation and identification; amateur radio communications; telemetry and telecommand services for aircraft and missiles; Digital Audio Broadcast; mobile satellite communication systems like Inmarsat and Iridium. It can be observed that the spectrum around the GNSS frequency bands is heavily used and thus unintentional 
Radio Frequency (RF) interference is likely to occur for a GNSS receiver at a low level. Naturally, also deliberate jamming of the GNSS signals can happen.

The topic of narrowband filtering has been investigated e.g. in [13-15]. In [13] and [15], notch filters are used for the detection and filtering of narrowband CWI in GNSS signals. In this paper we utilize a filtering technique that is adaptive and can detect and filter an unlimited number of CWIs. To study the effectiveness of the method, we compare the tracking Root Mean Square Error (RMSE) curves versus Signal-toInterference Ratios (SIRs), at different Carrier-to-Noise densities $\left(\mathrm{C} / \mathrm{N}_{0} \mathrm{~s}\right)$. The second section of this work presents the potential RF interferences in GNSS. The next section discusses the properties of the Binary-Offset-Carrier (BOC) modulation. The CWI filtering algorithm is presented in the next Section and the simulation setup is described in section 5. The results obtained from the Matlab simulations are illustrated in section 6 and the conclusions are presented in the last section.

\section{SOURCES OF INTERFERENCES IN GALILEO}

RF interferences can be classified after the width of the frequency spectrum used by the interference signal, comparative to the size of the bandwidth used by GNSS signal, in wideband or narrowband. The most restricted narrowband interference is a single frequency sine wave (Dirac impulse), called a CWI. The source of narrowband interferences can be found among the following: the surplus of energy from neighboring bands, located over or under the frequency of a GNSS carrier; by harmonics; by intermodulation products [2]. Harmonics are positioned at frequencies equal to integer multiples of the carrier frequency of an emitter and are produced by nonlinearities in the transmission stage. Intermodulation products may occur in the situation when signals of different frequencies are passed through a nonlinear device.

Air navigation is one of the most important applications of GNSS in the civilian domain. GNSS is able to optimize the efficient management of the airspace, can enhance air traffic safety and can lower air traffic costs. For this, GNSS services must provide [3]: accuracy, integrity, continuity and global availability. To improve the integrity of the navigation service, Galileo is planning to provide a Safety of Life (SoL) service in the E5a and E5b subbands of the L band. The service will offer the user a suitable notice of a faulty system status. In the last decades, DME employed the E5 subband. Fortunately this system is emitting pulses with a reduced duration $(3.6 \mu \mathrm{s})$, but the transmission power reaches high values, up to $2 \mathrm{~kW}$, compared to the transmitting power of approximate $100 \mathrm{~W}$ from a navigation satellite. There is also a major difference between the distances from these emitters to the receiver: a GNSS receiver aboard an aircraft can be as close as $0.1 \mathrm{~km}$ from an emitting DME station, but it will always be about $23200 \mathrm{~km}$ away from the emitter onboard the GNSS satellite. Thus it can be said that the reception of a GNSS signal can get very bad while a DME station is emitting its pulse signal. One technique of eliminating this interference caused by the DME is the "pulse blanking method" [9]. With this method the receiver input is turned off when a pulse is detected. The decrease of the positioning precision is not important because the duration of the pulse is quite short. Still, in the cases when signals from several DME stations are received, or when the air traffic is busy and numerous pulses are emitted, they become very frequent and the receiver must cut off the input 
often. As a result, the receiver will not be able to properly acquire and track the signal, because it lacks many samples [4].

\section{MODULATION AND DEMODULATION IN GNSS}

BOC modulation is a spread-spectrum modulation method that is currently employed in Galileo Open Services and GPS modernized signals. The Sine BOC (denoted to as BOC) modulation separates the signal spectrum in two components, symmetrically located around the carrier frequency. This is obtained by multiplying the pseudorandom (PRN) code with a rectangular subcarrier [6]. The usual symbolization is $\mathrm{BOC}\left(f_{s c}, f_{c}\right)$ or $\operatorname{BOC}(m, n)$, with $m=f_{s c} / f_{\text {ref }}$ and $n=f_{c} / f_{\text {ref }}$, where $f_{c}$ is the chip rate, $f_{s c}$ is the sub-carrier frequency and $f_{r e f}$ is the reference frequency (generally $f_{r e f}=1.023 \mathrm{MHz}$ ). The resulting split-spectrum signal shows good frequency sharing and in the same time presents simple implementation, good spectral efficiency, high precision, and optimized multipath resolution [6]. The BOC modulated signal $x(t)$ is the convolution between a BOC waveform $S_{B O C}(t)$ and a modulating waveform $d(t)$, as follows [9]:

$$
\begin{aligned}
& x(t)=\sum_{n=-\infty}^{+\infty} b_{n} \sum_{k=1}^{S_{F}} c_{k, n} S_{B O C}\left(t-n T_{s y m}-k T_{c}\right) \\
& =S_{B O C}(t) \otimes \sum_{n=-\infty}^{+\infty} \sum_{k=1}^{S_{F}} b_{n} c_{k, n} \delta\left(t-n T_{s y m}-k T_{c}\right) \\
& @ S_{B O C}(t) \otimes d(t)
\end{aligned}
$$

where $\oplus$ is the convolution operator, $d(t)$ is the spread data sequence, $b_{n}$ is the $n$th complex data symbol (in case of a pilot channel, it is equal to 1), $T_{\text {sym }}$ is the symbol period, $c_{k, n}$ is the $k$ th chip corresponding to the $n$th symbol, $T_{c}=1 / f_{c}$ is the chip period, $S_{F}$ is the spreading factor $\left(S_{F}=T_{s y m} / T_{c}\right)$, and $\delta(t)$ is the Dirac impulse. It is considered that $d(t)$ is a wideband data signal, spread by multiplication with a PRN sequence. The Power Spectral Density (PSD) of MBOC is a combination of BOC $(1,1)$ spectrum and $\mathrm{BOC}(6,1)$ spectrum. One method of producing the MBOC spectrum is that of utilizing Composite BOC (CBOC) time waveforms. The CBOC method is based on a weighted sum (or difference) of $\mathrm{BOC}(1,1)$ and $\mathrm{BOC}(6,1)$-modulated code symbols [9]. Three implementations of $\mathrm{CBOC}$ have been developed: $\mathrm{CBOC}(+), \mathrm{CBOC}(-)$ and $\mathrm{CBOC}(+/-)$. In this paper we will use the $\mathrm{CBOC}$ (minus) modulation, defined as [8]:

$$
\begin{gathered}
s_{C B O C(+)}(t)=w_{1} s_{B O C(1,1)}(t)+w_{2} s_{B O C(6,1)}(t) \\
s_{C B O C(-)}(t)=w_{1} s_{B O C(1,1)}(t)-w_{2} s_{B O C(6,1)}(t) \\
w_{1}=\sqrt{10 / 11} \quad w_{2}=\sqrt{1 / 11}
\end{gathered}
$$

The received GNSS signal passes through the RF front end and the intermediate frequency blocks. Afterwards it is downconverted and sampled and it is passed to the baseband stages of the receiver. Here, the code tracking loop tracks the phase of the PRN code of the incoming signal. This loop uses a Delay Lock Loop (DLL) which computes the correlation between the input signal and three replicas of the locally produced code. The three replicas (Early, Prompt and Late) are generated with an equal spacing between them. The replica that presents the highest correlation characterizes best the delay between the prompt replica and the received code. 
A code tracking method that is widespread is the Early-Minus-Late (EML). It employs a 0.5 chip spacing between the three local replicas. Because of the large spacing, the EML method does not need significant processing resources, but, in the same time, it has restricted multipath mitigation capability. The correlation lobes of the multipath replicas of the received signal can be falsely identified by the algorithm as the main correlation lobe of the received signal and, thus, positioning errors can occur. Therefore, several optimized EML-based methods have been introduced, especially to mitigate closely spaced multipath signals [10]. One of these enhanced EML methods, denoted as narrow EML (nEML) is based on the idea of strongly reducing the 1 chip spacing between the early and late correlators [11].

\section{ALGORITHMS FOR INTERFERENCE ELIMINATION}

In order to eliminate the CWIs, we employ an infinite impulse response notch filter, explained in [12]. The one chosen in this research is a second order filter and its $Z$ domain transfer function $H_{N}(z)$ is represented by the next equation:

$$
H_{N}(z)=\frac{1+\alpha}{2} \cdot \frac{1-2 \beta z^{-1}+z^{-2}}{1-\beta(1+\alpha) z^{-1}+\alpha z^{-2}}
$$

Parameters $\alpha$ and $\beta$ are defined as:

$$
\alpha=\frac{1-\tan (\beta / 2)}{1+\tan (\beta / 2)} \quad \beta=\cos \left(\omega_{N}\right), \omega_{N} \in[0, \pi]
$$

where $\omega_{N}$ is the angular notch frequency, defined as $\omega_{N}=2 \pi f_{c}$, with $f_{c}$ being the central frequency of the attenuating band of the filter. Parameter $\alpha$ controls the width of the -3 $\mathrm{dB}$ bandwidth. Its value is chosen at $\alpha=0.989$ for a minimum attenuation of the useful navigation signal. The purpose of parameter $\beta$ is to alter the central frequency of the notch filter.

The behavior of the filtering method (denoted as the minimum power method) is illustrated in the block diagram in Fig. 1. This is a time domain method, and thus it utilizes a relative reduced amount of computational resources and no Fourier transformations. The detection technique employed by this method, represented by the blocks with red colored margins in the diagram, consists in a loop that executes itself while CWIs are found. The detection method is widely described in [19].

The actual filtering takes place after determination of the frequencies of the interferences. The notch filter is applied successively to the input signal, using the central frequencies found during detection. After this filtering is over, the spectrum of the CBOC signal does not present any more CWIs, as it can be seen in the example plotted in Fig. 2. In the upper half of this figure, three CWIs have been added to the CBOC signal (the number of Dirac impulses is double in the figure because of the symmetry between the negative and positive frequencies). At the end of the detection and filtering stages, the spectrum of the signal does not present interferences, as it is illustrated in the lower half of the figure. 


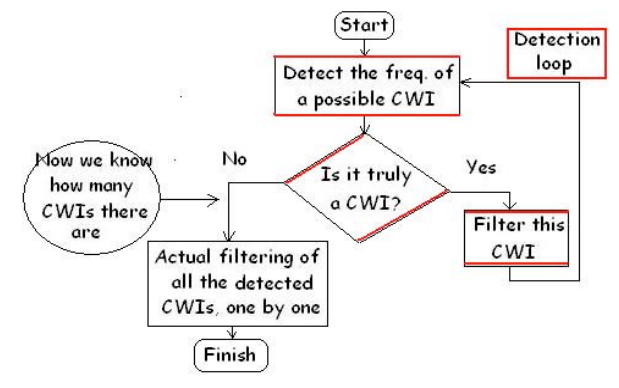

Fig. 1 Block diagram of the minimum power method

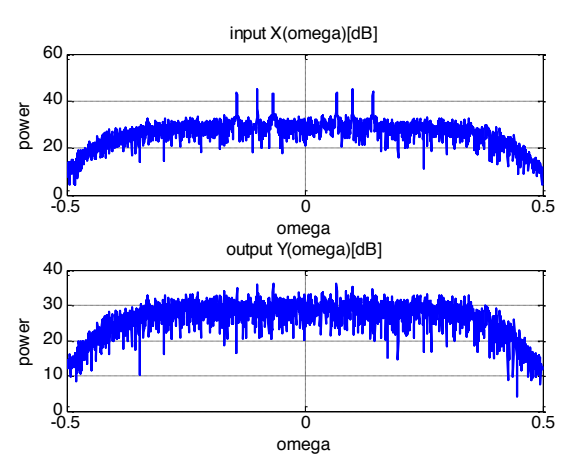

Fig. 2 Signal spectrum vs. angular frequency $\omega$

\section{DESCRIPTION OF THE SIMULATIONS}

To analyze the effect of CWI, we have simulated via Matlab the tracking of a CBOC signal which is corrupted by interference. The block diagram in Fig. 3 presents the principal components of the simulation setup. The CBOC signal explained earlier (with $f_{c}=1.023 \mathrm{MHz}$ ) is propagated through a radio channel modeled with AWGN. The CWI is modeled by a single-frequency, continuous sine wave, which is summed with the CBOC signal. As a result, a Dirac impulse is added over each of the two main lobes of the CBOC spectrum. We simulated a scenario with heavy interferences, with five simultaneous CWIs. Next, the resulting signal is fed to the filtering block. Afterwards, the filtered signal is processed by the tracking block, which employs the nEML algorithm.

The end result is represented by the curves of the Root Mean Square tracking Error versus SIR at several values of $\mathrm{C} / \mathrm{N}_{0}$. The performance of the method during the simulated scenario is examined by comparing the behavior of the RMSE as the value of SIR decreases.

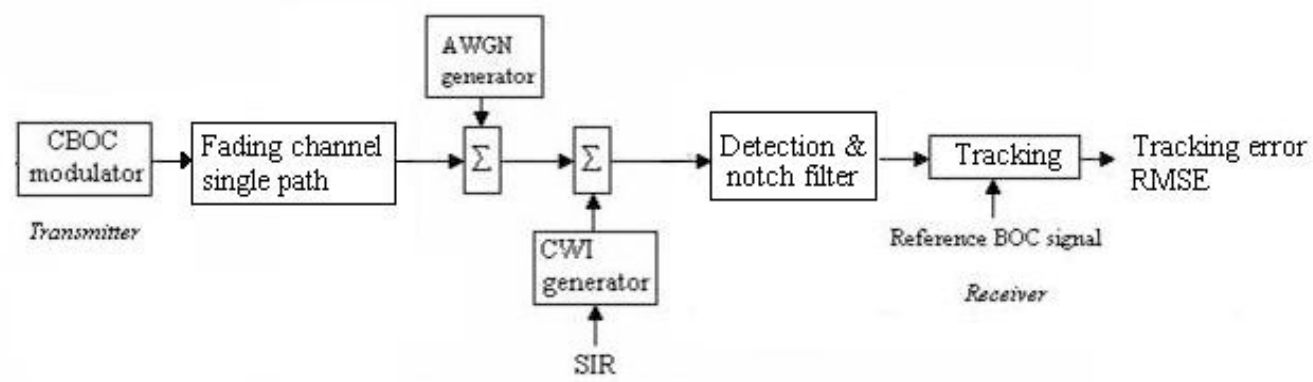

Fig. 3 Block diagram of the simulation setup 
Table 1 Values of the simulation parameters

\begin{tabular}{|l|l|}
\hline Parameter & Value \\
\hline Channel model & uncorrelated, \\
& Nakagami-m=0.8 \\
\hline Number of paths & 1 \\
\hline Oversampling factor & 4 \\
\hline Coh. integration time & $4 \mathrm{~ms}$ \\
\hline Noncoh. integration time & $1 \mathrm{~ms}$ \\
\hline Initial delay error & 0 chips \\
\hline
\end{tabular}

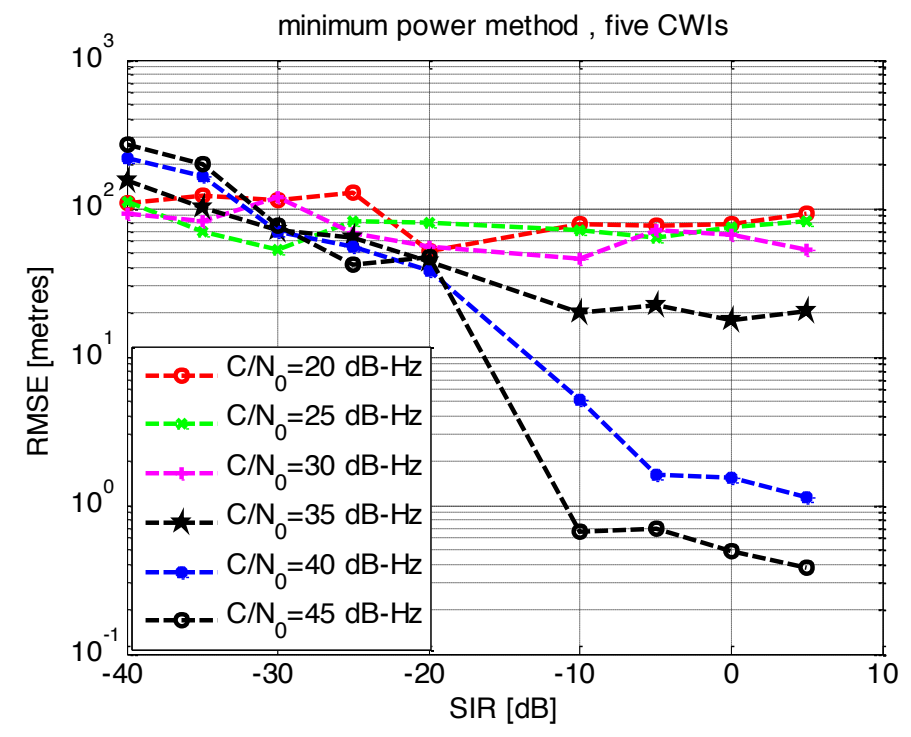

Fig. 4 Tracking RMSE vs. SIR for five CWIs, at various $\mathrm{C} / \mathrm{N}_{0}$

The simulation parameters are given in Table 1. SIR for one CWI is considered as:

$$
S I R_{d B}=20 \log _{10}\left(\frac{1}{A_{s}}\right)
$$

where $A_{S}$ is the amplitude of the sine (absolute value) and the amplitude of the CBOC waveform is normalized to 1 .

\section{RESULTS}

Extended simulations were run for a SIR covering the $[-40,5] \mathrm{dB}$ interval, with a $\mathrm{C} / \mathrm{N}_{0}$ in the $[20,45] \mathrm{dB}-\mathrm{Hz}$ interval. Lower values of the $\mathrm{C} / \mathrm{N}_{0}$ provided unsatisfactory results for the RMSE. As described earlier, we generated five CWIs, each with the same power, and added them over each of the main lobes of the CBOC spectrum. The five CWIs are spaced at $0.5 \mathrm{MHz}$. Fig. 4 presents the resulting variations of RMSE over the SIR interval. It can be observed that the tracking error decreases with the increase of the SIR and that the lowest error at high SIR is obtained at high $\mathrm{C} / \mathrm{N}_{0}$ values.

\section{CONCLUSIONS}

In this paper, we address the particular issue of filtering the CWIs affecting CBOC Galileo navigation signal, using an adaptive filtering technique. To study the effectiveness of the method, we compare the tracking RMSE curves versus SIRs, at different $\mathrm{C} / \mathrm{N}_{0} \mathrm{~s}$.

The results show that, at low SIR values, in the interval of $[-40,-30] \mathrm{dB}$, the tracking error is very high, (around $100 \mathrm{~m}$ ). The filtering method produces relatively good results (tracking error of less than $10 \mathrm{~m}$ ) at a SIR higher than $-15 \mathrm{~dB}$. Also it can be seen that the tracking error shows a quasi-constant value for low SIR and $\mathrm{C} / \mathrm{N}_{0}$ values, in the 
interval $[20,30] \mathrm{dB}-\mathrm{Hz}$. In these situations, the error is higher than $50 \mathrm{~m}$. If we compare the current results with the ones described in [19], it can be concluded that the increase of the number of CWIs, from three to five, degrades the performance of the filtering method, because the error increases in the plots when the number of CWIs increases (at the same $\mathrm{C} / \mathrm{N}_{0}$ ).

For further studies, the following topics seem to be interesting: studying the behaviour of other filtering methods in scenarios with heavy interferences, in comparison with the minimum power method; evaluation of the effect of more than 5 CWIs on the RMSE.

This work has been supported partly by the grant PN II Parteneriate 92-100/2008, 20082011, by Nokia Foundation Visiting Professor Grant, by the Academy of Finland, and by the Spanish Government project TEC2011-28219, which are gratefully acknowledged.

\section{REFERENCES}

[1] The European GNSS (Galileo) Open Service Signal in Space Interface Control Document, Issue 1.1, September 2010.

[2] E. D. Kaplan and C.J. Hegarthy, Understanding GPS Principles and application, Artech House, Boston, 2006, pp. 244-247.

[3] Civil Aviation Authority of the United Kingdom, CAA PAPER 2003/9 GPS Integrity and Potential Impact on Aviation Safety, April 2004.

[4] A. Steingass, A. Hornbostel, H. Denks, Airborne measurements of DME interferers at the European hotspot, DLR, in Proceedings of ENC GNSS, Naples, Italy, May 2009.

[5] F. Klinker and O.B.M. Pietersen, Interference of GPS signals: Influence of Licensed Transmitters on the GPS Signal Quality in the Netherlands' Airspace, National Aerospace Laboratory NLR, May 2000.

[6] J.W. Betz, The Offset Carrier Modulation for GPS Modernization, in Proc. of ION Technical Meeting, June 1999, pp. 639-648.

[7] E. S. Lohan, A. Lakhzouri and M. Renfors, Binary-Offset-Carrier Modulation Techniques With Application in Satellite Navigation Systems, Wireless Communications \& Mobile Computing, Vol. 7, 2007, pp. 767-779.

[8] J. Avila Rodriguez et al., CBOC- An Implementation of MBOC, First CNES Workshop on Galileo Signals and Signal Processing, Touluse, France, October 2006

[9] C. Hegarty et al., Suppresion of Pulsed Interference thorugh Blanking, Proceedings of the 56th Annual Meeting of The Institute of Navigation, San Diego, CA, USA, June 2000, pp. 399-408.

[10] M. Z. H. Bhuiyan, X. Hu, E.S Lohan, and M. Renfors, Multipath mitigation performance of multi correlator based code tracking algorithms in closed and open loop model, in Proc. of European Wireless Conference, May 2009, Aalborg, Denmak.

[11] A. J. V. Dierendonck, P. C. Fenton, and T. Ford, Theory and Performance of Narrow Correlator Spacing in a GPS Receiver, Journal of The Institute of Navigation, No. 39, Vol. 3, June 1992.

[12] Y.R. Chien, Y.C. Huang, D.N. Yang, and H.W. Tsao, A Novel Continuous Wave Interference Detectable Adaptive Notch Filter for GPS Receiver, GLOBECOM, Miami, USA, Dec 2010, pp.1-6. 
[13] D. Borio, L. Camoriano, L. Lo Presti, Two-Pole and Multi-Pole Notch Filters: a Computationally Effective Solution for Interference Detection and Mitigation, ITST, June 2007, pp.1-6.

[14] J. Vartiainen, J. Lehtomaki, H. Saarnisaari, M. Juntti, Limits of detection for the consecutive mean excision algorithms, Proceedings of CROWNCOM, June 2010, pp.15 .

[15] D. Borio, L. Camoriano, S. Savasta, S.; L. Lo Presti, Time-Frequency Excision for GNSS Applications, IEEE Systems Journal, Vol. 2, No.1, March 2008, pp.27-37.

[16] D. Cabric, S.M. Mishra, and R.W. Brodersen, Implementation issues in spectrum sensing for cognitive radios, in Proceedings of Asilomar Conf., pp. 772- 776, Nov. 2004.

[17] J. Goerlich et al., Signal analysis using spectral correlation measurement, Proc. of Instrumentation and Measurement Technology Conf., pp.1313-1318, May 1998

[18] M. Song, Characterizing cyclostationary features of digital modulated signals with empirical measurements using spectral correlation function, MSc Thesis, Air Force Institute of Technology, Ohio,USA, June 2011.

[19] A. Rusu-Casandra, E. S. Lohan, G. Seco-Granados, I. Marghescu, Investigation of Narrowband Interference Filtering Algorithms for Galileo CBOC Signal, in the ISI Conference Proceedings of the 3rd European Conference of Communications, Paris, France, pp. 274-279, Dec. 2012. 